\title{
Early results of integrated malaria control and implications for the management of fever in under-five children at a peripheral health facility: a case study of Chongwe rural health centre in Zambia Pascalina Chanda*1, Busiku Hamainza1, Susan Mulenga², Victor Chalwe ${ }^{3}$, Charles Msiska² and Elizabeth Chizema-Kawesha ${ }^{1}$
}

Address: ${ }^{1}$ Ministry of Health Headquarters, Lusaka, Zambia, ${ }^{2}$ Chongwe District Health Office, Ministry of Health, Lusaka, Zambia and ${ }^{3}$ Tropical Diseases Research Centre, Ndola, Zambia

Email: Pascalina Chanda* - pascychanda@yahoo.com; Busiku Hamainza - bhamainza@nmcc.org.zm; Susan Mulenga - suemm@ymail.com; Victor Chalwe - victorchalwe@yahoo.com; Charles Msiska - myekha@yahoo.com; Elizabeth Chizema-

Kawesha - elizabethchizema@yahoo.co.uk

* Corresponding author

Published: 17 March 2009

Malaria Journal 2009, 8:49 doi:10.1/86/1475-2875-8-49
Received: 28 August 2008

Accepted: 17 March 2009

This article is available from: http://www.malariajournal.com/content/8/I/49

(C) 2009 Chanda et al; licensee BioMed Central Ltd.

This is an Open Access article distributed under the terms of the Creative Commons Attribution License (http://creativecommons.org/licenses/by/2.0), which permits unrestricted use, distribution, and reproduction in any medium, provided the original work is properly cited.

\begin{abstract}
Background: Zambia has taken lead in implementing integrated malaria control so as to attain the National Health Strategic Plan goal of "reducing malaria incidence by $75 \%$ and under-five mortality due to malaria by $20 \%$ by the year $2010 "$. The strategic interventions include the use of long-lasting insecticide-treated nets and indoor residual spraying, the use of artemisinin-based combination therapies (ACT) for the treatment of uncomplicated malaria, improving diagnostic capacity (both microscopy and rapid diagnostic tests), use of intermittent presumptive treatment for pregnant women, research, monitoring and evaluation, and behaviour change communication. Financial barriers to access have been removed by providing free malaria prevention and treatment services.

Methods: Data involving all under-five children reporting at the health facility in the first quarter of 2008 was evaluated prospectively. Malaria morbidity, causes of non-malaria fever, prescription patterns treatment patterns and referral cases were evaluated

Results: Malaria infection was found only in $0.7 \%$ (10/I378), I.8\% (25/378) received anti-malarial treatment, no severe malaria cases and deaths occurred among the under-five children with fever during the three months of the study in the high malaria transmission season. $42.5 \%$ (586/1378) of the cases were acute respiratory infections (non-pneumonia), while 5.7\% (79/1378) were pneumonia. Amoxicillin was the most prescribed antibiotic followed by septrin.

Conclusion: Malaria related OPD visits have reduced at Chongwe rural health facility. The reduction in health facility malaria cases has led to an increase in diagnoses of respiratory infections. These findings have implications for the management of non-malaria fevers in children under the age of five years.
\end{abstract}




\section{Background}

Malaria accounts for $50 \%$ of outpatient attendances and $20 \%$ admissions in Africa [1]. In sub-Saharan Africa alone, annual malaria deaths are estimated at 1.3 million for all age groups. This has resulted into high expenditures on malaria control. WHO estimates show that countries may spend more than $40 \%$ of their health budget on malaria treatment and prevention [2]. This increase in health care expenditure and the negative effects of malaria on productivity slows down economic growth for these countries [3]. Not only does malaria have a negative impact on economic growth at national level [4], but it also affects the income levels of poor households [5]. Thus, integrated malaria control is high on the health agenda, as described in the Abuja declaration of 2000 [6].

In Zambia, malaria accounts for about 4 million cases (confirmed and unconfirmed) with approximately 6,400 deaths reported at health facilities country-wide [7]. The case fatality rates among hospital admissions are estimated to be $40 / 1,000$ [8]. It is for this reason that malaria has taken centre stage in the National Health Strategic Plan (2006) and the fifth National Development Plan (2006). The main goal for malaria control is "to reduce malaria incidence by $75 \%$ and under-five mortality due to malaria by $20 \%$ by the year 2010" [9].

In order to achieve these goals, the Ministry of Health through the national malaria control programme is implementing integrated malaria control. The strategic interventions include the use of long-lasting insecticidetreated nets and indoor residual spraying, the use of artemisinin-based combination therapies (ACT) for the treatment of uncomplicated malaria, improving diagnostic capacity (both microscopy and rapid diagnostic tests), encouraging the use of intermittent presumptive treatment for pregnant women, research, monitoring and evaluation, and behaviour change communication. Financial barriers to access have been addressed, all malaria services (treatment and prevention) are offered free-of-charge. In April 2006, the government removed user fees in all 56 rural districts of Zambia [10].

All these interventions are known to be effective tools for malaria control. Further, studies in South Africa showed that ACT has the potential to reduce expenditure on malaria treatment [11]. Studies conducted elsewhere have demonstrated that both IRS and ITNs are effective prevention strategies in various settings [12-14]. In country studies have shown that ACT is a cost-effective treatment for malaria $[15,16]$. Utzinger and colleagues demonstrated that integrated malaria control resulted into better health for the community and higher productivity for the mining sector on the copperbelt in Zambia [17].
The Malaria indicator survey for 2008 report indicates that $75 \%$ Zambians own at least one ITN, 62\% women received two or more doses of IPT, more than $77 \%$ of households in IRS designated districts were sprayed $[18,19]$. Further, in a health facility survey conducted in 104 health facilities in Zambia, it was found that $60 \%$ of the health facilities used ACT (artemether-lumefantrine), $73 \%$ had diagnostic capacity and at least $42 \%$ of children with uncomplicated malaria were treated with ACT [20]. An assessment on the impact of user fees removal on health facility utilisation found that from 2006 to 2007, there was an increase in utilisation of rural health facilities in the order of $7 \%$ and $55 \%$ for under-five and over-five years populations respectively [10]. Thus, both the population and health facility surveys have shown that malaria control interventions are reaching the service delivery points and the community members are having access. The malaria control programme has received financial and technical support from various multilateral, bilateral and private organisations.

In as much as evidence on process and outcome indicators exists, there has been less documentation of the impact of scaling-up malaria control in Zambia. As a result, a prospective study on the impact of scaling up malaria control is being conducted in various sites. This paper presents a case study of one particular rural district in Zambia where malaria control has began to show positive results at health facility level.

\section{Methods \\ Study sites}

The study was conducted in Chongwe district at Chongwe rural referral health centre. Chongwe district is located 35 $\mathrm{Km}$ from Lusaka city. This rural district has a population of 170,943 inhabitants. Most of the inhabitants are subsistence farmers. The district was among the first seven districts to pioneer use of ACT in 2003. In 2006, 31,322 people from 6,417 households were protected by IRS, while in 2007 the number of people protected with IRS was 63,307 (15,398 households). The total number of ITNs distributed in 2007 was 26,603. Chongwe rural health centre is a sentinel site for malaria information system and drug efficacy monitoring. The catchment area for Chongwe has also been selected as a site for demographic site surveillance.

\section{Study design and population}

The study is part of a larger prospective evaluation of the impact of scaling up malaria control on health facility disease morbidity and mortality. All children aged five years and below who reported to Chongwe rural health facility with fever (or history of fever) from December 2007 to February 2008 were included in the study. All the children were screened by either a nurse or clinical officer on duty. 
Confirmation of malaria infection was performed by either RDT or microscopy, when RDT was used, a microscopy slide was taken for validation. Treatment of malaria patients was based on the treatment guidelines for malaria in Zambia [21].

The health workers involved in patient management included a nurse, a clinical officer, a laboratory technologist, dispenser and information officer. A community health worker was always available to assist with patient tracking and informed consent. The site was supervised by a team of two scientific officers and two medical doctors from Central and district level.

Non-malaria fevers were further investigated based on standard treatment guidelines for Zambia. All the drugs were supplied through routine mechanisms (Central Medical Stores) based on the essential medicines list for the country.

\section{Study procedures}

The health workers at the health facility received an orientation prior on the commencement of the study on malaria treatment guidelines. Emphasis was placed on management of fever in children and more so the importance of management of non-malaria fevers. The district clinical care specialist and the health information officer were part of the team trained for the study. This was to enable them provide onsite supervision for both patient management and data management. No other interventions were applied.

All under-five children reporting to the out-patient department (OPD) of the health centre were identified and referred to the study screening room. A clinical officer or nurse then assessed the patient and performed a RDT test. Further management of the patient was based on the test result of the RDT. A microscopy slide was then taken for comparison reading by a laboratory technician on site. A sample of the slides was then taken to central level for quality assurance.

The patients were followed up on days 3, 7 and 14 . The mothers were then requested to return to the health facility should the child develop any illness after that. Community health workers continued follow-up in the community to ensure that the children were not kept at home in case they fell sick.

\section{Treatment}

The health facility receives a health centre drug kit from central medical stores. The drug kit contains all the drugs and supplies required for health service delivery for a second level health centre. All drugs are provided free to the patients. This is because the basic health care package in
Zambia is free and, further, all rural health centres do not charge user fees as per policy recommendation [10]. The health worker decision on what medicine to prescribe to the children was largely based on their training and the stipulations in the standard treatment guidelines and depending on drug availability.

A medical doctor provided supervision on clinical aspects of patient management, a biomedical scientist supervised diagnostic procedures and the scientific officers provided oversight on the smooth running of the project and adherence to complete and correct reporting of all patients.

All data on patients age, complaint, diagnosis and prescription was recorded in the patient registers. The laboratory also kept a daily log sheet of all patients seen at the laboratory. The treatment outcome was finally recorded once the patient reached the endpoint in the study.

Retrospective parasite data for the facility from 2003 to 2008 was also reviewed in order to understand trends in parasite prevalence over the years.

\section{Data entry and analysis}

The data was entered and analysed in Microsoft Office Excel 2007. Data was entered by data clerks with a clinical background to ensure that all information was well entered. The clinical officer and scientific officer provided supervision to the data entry clerk. The entries were verified and corrections made on a record by record basis. Frequencies were generated for the parameters under investigation. The analysis included generation of disease burden, prescription practices and patient treatment outcome.

The study was approved by the Ethics Committee of the Tropical Disease Research Centre. Patient data was recorded by study numbers. All information was kept by the project staff. However, the study was also obliged to provide accurate reports to the facility HMIS to ensure that no cases were missed when the facility was reporting on the indicators required.

\section{Results \\ Malaria burden}

The number of children under five who sought care from the facility between December 2007 and February 2008 was 1,383 . These children came from the different regions of the catchment area of Chongwe rural health centre in Chongwe district. However, five children were excluded from analysis as there was no record of their reason for visiting the health facility, leaving a total analysable sample of 1,378 . The average age for children visiting the health facility was 20.4 months (about one year and six months). The average temperature for all visits was $38.0^{\circ} \mathrm{C}$. 
Of the 1,378 children under five years, who were enrolled or visited the health facility, $0.7 \%(10 / 1378)$ were found positive for malaria and treated with artemether-lumefantrine (the first-line treatment for Zambia). 15 children who were negative for malaria were also treated with antimalarials (13 received artemether-lumefantrine, while two received sulphadoxine-pyrimethamine). The two children who were treated with SP were three and seven months old, respectively. Thus, $1.8 \%$ children were treated with an anti-malarial $(25 / 1378)$ regardless of their malaria status. The average temperature for patients with malaria was $39.0^{\circ} \mathrm{C}$ (range $37.5^{\circ} \mathrm{C}$ to $40.2^{\circ} \mathrm{C}$ ) and the average age was 30 months (range 12 to 58 months).

A sample of 200 slides was taken for validation by two independent laboratory technicians. There was no disagreement with the result of the test for both positive and negative rapid diagnostic test result. The RDT used was the histidine-rich protein II (HRP-2) type, ICT Mal Pf. by ICT Diagnostics of South Africa.

Microscopy examination of the 10 positive slides did not show any mixed infections, only mono infections with Plasmodium falciparum were detected. Further, none of the patients who reported to the health facility had severe malaria and no deaths due to malaria were reported during the study period. Table 1 outlines the monthly visits and malaria parasite rates. More visits were reported in January due to high cases of skin infections and nonbloody diarrhoea in three areas of Chongwe (Dam area, Township and Libuko) in January alone.

\section{Causes of outpatient visits}

Acute respiratory infections (ARI) non-pneumonia $(42.5 \%)$ and pneumonia (5.7\%) were among the top five causes of morbidity at the facility. Malaria was not even among the top 10 causes of visitation at this facility (ranked number $15(0.7 \%)$. Among the least causes of morbidity were diseases such as one case of tuberculosis (29 months) and one case of chicken pox was reported in the three months. Fever of unknown origin was found in 20 patients $(1.5 \%)$, however, these patients were treated with paracetamol and no further complaints were received from parents or caretakers within the 14 days of follow up. Another 34 children (2.5\%) were found not to have any illness and therefore did not need to visit the facility at all. However, they were treated with either paracetamol or some antibiotics.

\section{Referrals}

A total of five children were referred for further management to the University Teaching Hospital. The referrals included one each for skin infection, severe malnutrition, fractured arm, conjunctivitis and the only case of tuberculosis. Three children were locally admitted at the health centre, two for pneumonia and one for severe diarrhoea.

\section{Treatment characteristics}

Piriton was mostly prescribed than all the treatments as shown in table 2. Amoxyl was the most prescribed antibiotic $(20 \%)$, followed by septrin $(10.5 \%)$ and pen $\mathrm{V}$ (9.1\%). Anti-malarials were prescribed in less than $2 \%$ of the patients, 23 patients were treated with Coartem ${ }^{\circledast}$ (artemether-lumefantrine) and two with Fansidar ${ }^{\circledR}$ (SP). All the patients treated with anti-malarials were not prescribed an antibiotic, but paracetamol as per standard treatment guidelines for patients with no concomitant illness. Other drugs included treatment for burns, sprain, worms, etc.

About 23.4\% (323/1378) of all patients were prescribed with a single drug only. Among the 323 patients who received a single drug, $35 \%$ were treated with paracetamol alone, followed by Piriton ${ }^{\circledast}$, Oral Rehydration Salts (ORS) and Amoxy $^{\circledR}$ respectively as shown in Table 3. Five patients were treated with Coartem ${ }^{\circledast}$ only $(1.5 \%)$.

It was also observed that $77 \%$ of all the patients were prescribed at least two drugs. Paracetamol and a given treatment was a common combination accounting for $79 \%$ of the double prescriptions (prescriptions of two drugs). None of the patients were prescribed more than one antibiotic. However, it is possible that some patients may have been prescribed paracetamol in addition to the two antibiotics and this may not have been documented.

\section{Trends in parasite prevalence among under-five children with fever at OPD}

The parasite prevalence among fever patients has been reducing from a high of $83 \%$ in 2004 to $0.7 \%$ in 2008 [22]. Further, population surveys have also shown that

Table I: Facility visits for and malaria treatment

\begin{tabular}{|c|c|c|c|}
\hline Month & Number of visits for Under-five & Number positive (Parasite prevalence) & Total anti-malarial treatments \\
\hline December 2007 & 378 & $\begin{array}{c}2(0.53 \%) \\
95 \% \mathrm{Cl}[0.2-1.9]\end{array}$ & $\begin{array}{c}6(1.6 \%) \\
95 \% \mathrm{Cl}[0.7-3.4]\end{array}$ \\
\hline January 2008 & 638 & $\begin{array}{c}7(1.1 \%) \\
95 \% \mathrm{Cl}[0.5-2.2]\end{array}$ & $\begin{array}{c}13(2.0 \%) \\
95 \% \mathrm{Cl}[1.2-3.4]\end{array}$ \\
\hline February 2008 & 362 & $\begin{array}{c}\mathrm{I}(0.3) \% \\
95 \% \mathrm{Cl}[0.01-1.5]\end{array}$ & $\begin{array}{c}6(1.7 \%) \\
95 \% \mathrm{Cl}[0.8-3.4]\end{array}$ \\
\hline Total visits & 1378 & $\begin{array}{c}10(0.7 \%) \\
95 \% \mathrm{Cl}[0.4-1.3]\end{array}$ & $\begin{array}{c}25(1.8 \%) \\
95 \% \mathrm{Cl}[1.2-2.7]\end{array}$ \\
\hline
\end{tabular}


Table 2: Proportion of patients treated with a named drug

\begin{tabular}{lcc}
\hline Treatment & Number & Frequency (\%) \\
\hline Piriton & 396 & 28.7 \\
Amoxyl & 274 & 19.9 \\
ORS & 151 & 11.0 \\
Septrin & 145 & 10.5 \\
Pen V & 125 & 9.1 \\
Panadol & 114 & 8.3 \\
Flagyl & 48 & 3.5 \\
Coartem & 23 & 1.7 \\
Hydrocortisone & 22 & 1.6 \\
Nystatin & 17 & 1.2 \\
Phenergan & 13 & 0.9 \\
Cephalexin & 10 & 0.7 \\
Erythromycin & 9 & 0.7 \\
Clotrimazole & 8 & 0.6 \\
Procaine penicillin & 7 & 0.5 \\
Other & 16 & 1.2 \\
\hline Total & 1378 & \\
\hline
\end{tabular}

there has been a reduction in parasite prevalence among the under-five population from household surveys from $20 \%$ in 2006 to $11 \%$ in $2008[18,19]$.

\section{Discussion}

The study has shown parasite rates lower than the national average. The health facility is situated in a district where an integrated package for malaria control has been implemented. The parasite prevalence at OPD was less than $1 \%$, only 10 confirmed cases in three months during the rainy season. Earlier parasite survey results conducted at the same facility in January and February 2006 among under-five children, recorded a parasite prevalence of $28 \%$

Table 3: Characteristics of single primary prescriptions

\begin{tabular}{lcc}
\hline Single prescriptions & Number & $\%$ \\
\hline Panadol & 114 & 35.3 \\
Prirton & 63 & 19.5 \\
ORS & 40 & 12.4 \\
Amoxyl & 36 & 11.1 \\
Pen V & 23 & 7.1 \\
TEO & 14 & 4.3 \\
Septrin & 12 & 3.7 \\
Phernergan & 5 & 1.5 \\
Coartem ${ }^{\circledR}$ & 5 & 1.5 \\
Cefalexin & 4 & 1.2 \\
Ciproflaxin & 4 & 1.2 \\
TSO & 1 & 0.3 \\
X-Pen & 1 & 0.3 \\
Griseofulvin & 1 & 0.3 \\
\hline Total & 323 & \\
\hline
\end{tabular}

at OPD, 7.9\% among pregnant women and $7.0 \%$ among asymptomatic primary school children (aged 2-14 years) at Chongwe basic school [23]. Further, in 2005, out of the 3338 all OPD visits, malaria was confirmed in $35 \%$ of all patients with fever, while in 2006, malaria accounted for $24 \%$ of health facility visits at Chongwe rural health facility [24]. So it appears that the malaria disease burden at Chongwe rural health facility has been on a steady decline as shown in figure 1 .

The availability of diagnostic facilities (either RDT or microscopy) created an opportunity to correctly identify malaria and non-malaria cases. This is an important step in improving not only malaria management, but overall patient management. The training which was given to the health workers before the study seemed to have assisted with using the diagnostic results to inform decision to prescribe an anti-malarial. Previous studies in Zambia showed that health workers still prescribed anti-malarials to more than $50 \%$ of negative cases [25].

Respiratory infections were diagnosed in more than $40 \%$ of the children under five years of age during the study period. It was not possible in this study to assess the quality of these diagnoses which were made for non-malaria fevers. However, the supervision reports for medical supervisors showed that patients were managed as per standard treatment guidelines. As malaria-related fever are reduced, the management of fever in under-five children is an area of further research to help identify gaps or document best practices where these may exist. This also has implications for home-management of fever using community health workers. The management of malaria and pneumonia studies are a necessary area for implementation research involving community health workers.

As seen from the prescription practices in this study, the use of antibiotics will increase to replace over prescription of anti-malarials at health facility level. Therefore, systematic antimicrobial resistance monitoring to these drugs should be developed. However, it was satisfying to note that paracetamol was prescribed in cases which were found not to need any medication as opposed to the use of antibiotics.

This study has also demonstrated that improved case management reduces severe malaria or severe patient outcomes. The health facility had effective first line treatment as per treatment policy in Zambia and diagnostic tools were readily available not only to the laboratory technician, but also to the nurses in the screening room. There was no severe malaria and no deaths reported among all the under-five children. Further, none of the referrals were malaria related. The community health worker was very useful in patient follow ups and feedback to the facility. 


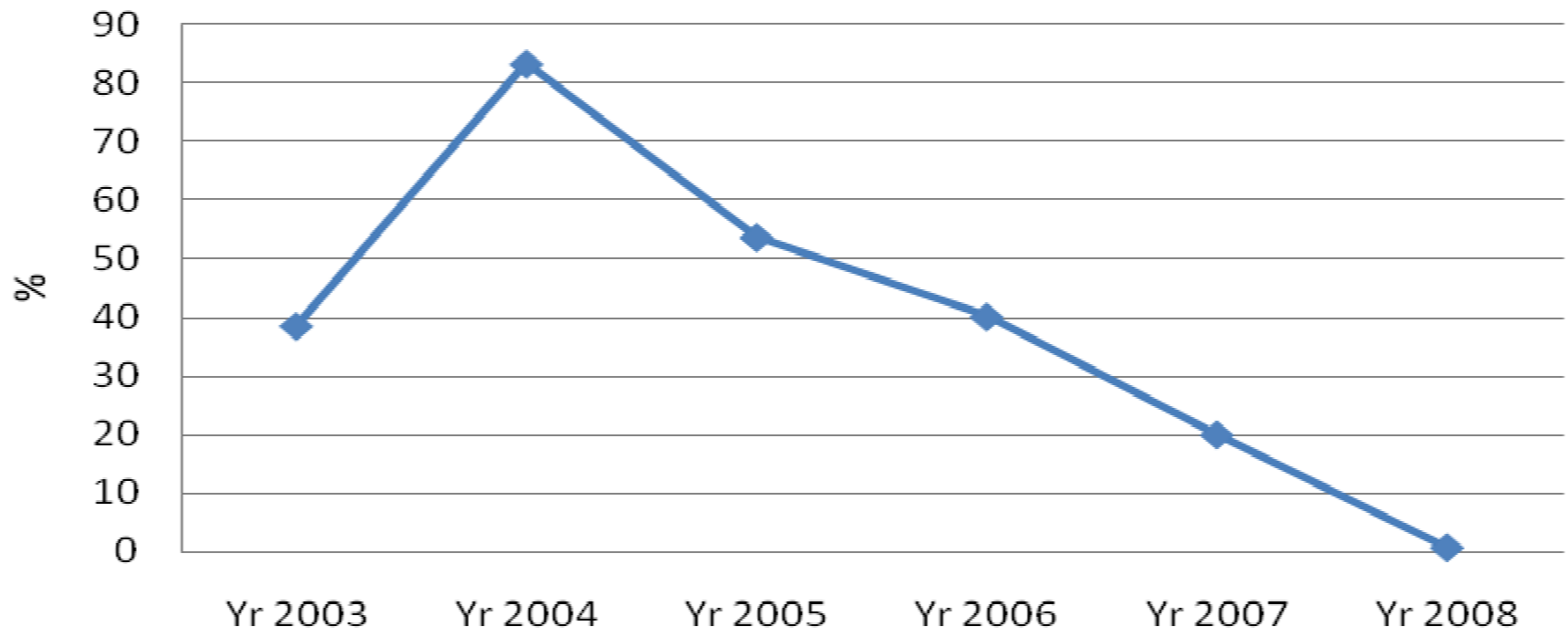

Figure I

Trends in parasite rates among fever patients at Chongwe health facility 2003-2008.

Some of the observation from this study is that the health system stands to potentially benefit from integrated malaria control through reduced malaria related revisits, reduced referrals, reduction in anti-malarial needs and in the long run reduction in health worker work load. However, the magnitude of these benefits need to be quantified by economic studies. Other studies in Africa have shown that rapidly scaling up coverage of effective treatment and vector control reduces malaria morbidity and mortality as shown in South Africa [26] and Zanzibar [27].

The study findings also seem to challenge the widely used indicator: "proportion of children with fever who received an anti-malarial". From this study, one could see that very few patients with fever were prescribed an anti-malarial because they really did not need to. So the indicator as it stands is of little use for programme managers and district personnel. However, it is important to measure among the "true malaria" cases, how many received an antimalarial (or ACT). This will be important as more and more diagnostics are being scaled-up.

Implementing integrated malaria control implies investing in changing health worker prescription practices based on evidence based development of treatment guidelines.

The study's main limitation is the lack of assessment of the accuracy of non-malaria diagnoses. However, as can be seen from the study there were no adverse health outcomes.

\section{Conclusion}

Implementing integrated malaria control has the potential to reduce malaria related visits at the health facility. However, respiratory infections are still prevalent. These findings have implications for the management of nonmalaria fevers in children under the age of five years. Management of fever in under-five children needs updating based on the changes in epidemiology. As malaria will no longer be the suspect, potential mismanagement of nonmalaria fevers is an important area of research. Improved health facility surveillance will be useful in redefining disease priorities in a given locality.

\section{Competing interests}

The authors declare that they have no competing interests.

\section{Authors' contributions}

PC designed the study protocol, supervised data collection, analysis and drafted the manuscript. BH was part of the protocol development, field supervision, data entry and provided input during manuscript development. VC trained field staff, supervised field work and participated in manuscript development. ECK $s$ revised the protocol, supervised the field work and provided input in the manuscript development. CM supervised data collection, clinical management and participated in the manuscript development. SM participated in data collection, supervised data management and provided input on the manuscript.

\section{Acknowledgements}

The funding was made possible through the Global Fund for HIVIAIDS,

Tuberculosis and Malaria grant for Round I, Phase 2, Year 3 for Zambia. 
We are grateful for all the commitment and support of the project staff and the district and all the caretakers and mothers who supported the study $100 \%$. Finally, we thank the Ministry of health for ensuring that funds were disbursement on time and for supporting the study.

\section{References}

I. World Health Organization: Africa Malaria Report WHO Regional offices for Africa and Eastern Mediterranean; 2006.

2. World Health Organization: Global RBM Online Database 2007 [http:/ /www.who.int/globalatlas/default.asp].

3. Sachs J, Malaney P: The economic and social burden of malaria. Nature 2002, 4I 5:680-685.

4. Gallup FL, Sachs J: The economic burden of malaria. Am J Trop Med Hyg 200I, 64:85-96.

5. Chuma J, Gilson L, Molyneux C: Treatment-seeking behaviour, cost burdens and coping strategies among rural and urban households in Coastal Kenya: an equity analysis. Trop Med Int Health 2007, I 2:673-86.

6. World Health Organization: The African Summit on Roll Back Malaria, Abuja, April 25, 2000 Geneva, Switzerland, World Health Organization. Roll Back Malaria; 2000.

7. Ministry of Health: Health Management Information System. Lusaka. Zambia 2007.

8. National Malaria Control Centre: Annual Report for Malaria Control. Lusaka. Zambia 2007.

9. National Malaria Control Programme: National Malaria Strategic Plan 2006-20 I 0. Lusaka, Zambia 2006.

10. Masiye F, Chitah BM, Chanda P, Simeo F: Removal of user fees at Primary Health Care facilities in Zambia: A study of effects on utilisation and quality of care. EQUINET Discussion Paper series 57 EQUINET, UCT HEU: Harare; 2007.

II. Muheki C, Mclntyre D, Barnes KI: Artemisinin-based combination therapy reduces expenditure on malaria treatment in KwaZulu Natal, South Africa. Trop Med Int Health 2004, 9:959-966.

12. Bhatia MR, Fox-Rushby J, Mills A: Cost-effectiveness of malaria control interventions when malaria mortality is low: insecticide-treated nets versus in-house residual spraying in India. Soc Sci Med 2004, 59:525-39.

13. Conteh L, Sharp BL, Streat E, Barreto A, Konar S: The cost and cost-effectiveness of malaria control by residual housespraying in southern Mozambique: a rural and urban analysis. Trop Med Int Health 2004, 9:125-132.

14. Goodman CA, Mnzava AE, Dlamini SS, Sharp BL, Mthembu DJ, Gumede JK: Comparison of the cost and cost effectiveness of insecticide treated bednets and residual-house spraying in KwaZulu-Natal, South Africa. Trop Med Int Health 200I, 6:280-295.

15. Chanda P, Masiye F, Chitah BM, Sipilanyambe N, Hawela M, Banda P, Okorosobo $\mathrm{T}$ : A cost-effectiveness analysis of artemethelumefantrine for treatment of uncomplicated malaria in Zambia. Malar J 2007, 6:21.

16. Chanda $\mathrm{P}$, Sikaala CH, Kapelwa W, Moonga H, Njunju E, Macdonald $M$, Thea $D$, Hamer $D H$, Sipilanyambe $N$ : Assesment of the therapeutic efficacy of artemether-lumefantrine (Coartem ${ }^{\circledR}$ ) and sulphadoxine-pyrimethamine (SP)-artesunate in Zambian children. Proceedings of the 53rd Annual Meeting of the Society of Tropical Medicine and Hygiene, 7 - I I November 2004, Miami, FL 2004.

17. Utzinger J, Tozan Y, Doumani F, Singer BH: The economic pay off of integrated malaria control in the Zambian Copperbelt between 1930 and 1950. Trop Med Int Health 2002, 8:657-677.

18. Ministry of Health: Malaria Indicator Survey. Lusaka, Zambia 2006.

19. Ministry of Health: Malaria Indicator Survey. Lusaka, Zambia 2008.

20. Zurovac D, Ndhlovu M, Sipilanyambe N, Chanda P, Hamer DH, Simon JL, Snow RW: Paediatric malaria case management with artemether-lumefantrine in Zambia: a repeat cross sectional study. Malaria Journal 2007, 6:31.

21. National Malaria Control Centre: Guidelines for the Diagnosis and Treatment of Malaria in Zambia. Central Board of Health. Lusaka 2004.

22. Ministry of Health: Assessment of the therapeutic efficacy of antimalarials 2003-2007. Lusaka, Zambia 2007.

23. Chanda P, Chanda E, Hawela MB, Chizema EK: Baseline parasitological assessments and accuracy of rapid diagnostic tests in districts implementing indoor residual spraying for malaria prevention in Zambia. Zambia Medical Journal 2007, 34:2.

24. Chanda P: Cost and Cost-effectiveness Analysis of the Available Strategies for Diagnosing Malaria in Outpatient Clinics in Zambia. In Masters Dissertation Health Economics Unit, University of Cape Town; 2006.

25. Hamer DH, Ndhlovu M, Zurovac D, Fox M, Yeboah-Antwi K, Chanda $P$, Sipilinyambe N, Simon JL, Snow RW: Improved diagnostic testing and malaria treatment practices in Zambia. JAMA 2007, 297:2227-2231.

26. Barnes KI, Durrheim DN, Little F, Jackson A, Mehta U, Allen E, Dlamini SS, Tsoka J, Bredenkamp B, Mthembu DJ, White NJ, Sharp BL: Effect of artemether-lumefantrine policy and improved vector control on malaria burden in KwaZulu-Natal, South Africa. PLoS Medicine 2:e330.

27. Bhattarai A, Ali AS, Kachur SP, Mårtensson A, Abbas AK, Khatib R, Al-Mafazy AW, Ramsan M, Rotllant G, Gerstenmaier JF, Molteni F, Abdulla S, Montgomery SM, Kaneko A, Björkman A: Impact of artemisinin-based combination therapy and insecticidetreated nets on malaria burden in Zanzibar. PLoS Medicine 4:e309.

Publish with Bio Med Central and every scientist can read your work free of charge

"BioMed Central will be the most significant development for disseminating the results of biomedical research in our lifetime. "

Sir Paul Nurse, Cancer Research UK

Your research papers will be:

- available free of charge to the entire biomedical community

- peer reviewed and published immediately upon acceptance

- cited in PubMed and archived on PubMed Central

- yours - you keep the copyright 